\title{
Genetic and Environmental Influences on Correlations Between Hearing and Cognitive Functions in Middle and Older Chinese Twins
}

\author{
Chunsheng $\mathrm{Xu},{ }^{1,2,3}$ Dongfeng Zhang, ${ }^{1}$ Xiaocao Tian, ${ }^{2,3}$ Haiping Duan, ${ }^{1,2,3}$ Yili Wu, ${ }^{1}$ \\ Zengchang Pang, ${ }^{1,2,3}$ Shuxia $\mathrm{Li}^{4}{ }^{4}$ and Qihua Tan ${ }^{4,5}$ \\ ${ }^{1}$ Department of Epidemiology and Health Statistics, School of Public Health, Qingdao University, Qingdao, Shandong, \\ China \\ ${ }^{2}$ Qingdao Municipal Center for Disease Control and Prevention, Qingdao, Shandong, China \\ ${ }^{3}$ Qingdao Institute of Preventive Medicine, Qingdao, Shandong, China \\ ${ }^{4}$ Unit of Human Genetics, Department of Clinical Research, University of Southern Denmark, Odense, Denmark \\ ${ }^{5}$ Epidemiology and Biostatistics, Department of Public Health, University of Southern Denmark, Odense, Denmark
}

\begin{abstract}
The genetic and environmental impacts on correlations between hearing and cognitive functions have not been well studied. Cognitive function was evaluated by the Montreal Cognitive Assessment (MoCA). Hearing function was assessed by audiometric pure-tone hearing thresholds at different frequencies, including $0.5 \mathrm{kHz}, 1 \mathrm{kHz}, 2 \mathrm{kHz}, 4 \mathrm{kHz}, 8 \mathrm{kHz}$, and $12.5 \mathrm{kHz}$, with the lower hearing thresholds indicating better hearing function. Cognitive and hearing functions were measured on 379 complete twin pairs (240 monozygotic and 139 dizygotic pairs) with a median age of 50 years (range: $40-80$ years). Bivariate twin models were fitted to quantify the genetic and environmental components of the correlations between hearing and cognitive functions. The analysis showed significantly high genetic correlation between $2 \mathrm{kHz}$ of hearing and cognition $\left(r_{\mathrm{G}}=-1.00,95 \% \mathrm{Cl}[-1.00,-0.46]\right)$ and moderate genetic correlation between $4 \mathrm{kHz}$ of hearing and cognition $\left(r_{\mathrm{G}}=-0.62,95 \% \mathrm{Cl}[-1.00,-0.14]\right)$. We found no significant genetic correlations between low as well as high frequencies of hearing and cognition. Low to moderate common and unique environmental correlations were shown between low frequencies of hearing and cognition $(-0.13$ to -0.39$)$ and the common environmental correlation between $8 \mathrm{kHz}$, one of the high frequencies of hearing, and cognition (-0.22). The middle frequencies of hearing and cognitive functions may have a shared genetic basis. Low frequencies of hearing and cognition may share similar common and unique environmental factors. At $8 \mathrm{kHz}$, the high frequency of hearing and cognition may share similar common environment. This twin study detected a significant genetic and environmental basis in the phenotype correlation between cognition and hearing, which differed across frequencies.
\end{abstract}

Keywords: aging, hearing, cognitive function, genetic correlation, Chinese twins

Population aging is a major global public health and social problem. Human aging is accompanied by a general trend of poor functional performances and slowing of mental processing, affecting multiple functional profiles, including hearing and cognitive changes. Aging-related hearing and cognitive impairment can result in reduced quality of life, frustration, social isolation, and dependence, and can also affect healthy people, especially those who have family members who are impaired. It is thus an essential aim of research in order to promote healthy aging.

Aging-related hearing loss is the most common sensory deficit in the older population (Huang \& Tang, 2010). The auditory system, including low, middle, and high frequencies of hearing, is essential to conduct communication and enable speech understanding. Good hearing ability can also help to detect, identify, and localize sound. Poor hearing may lead to misunderstandings and communication errors,

RECEIVED 10 May 2017; ACCEPTED 27 June 2017. First published online 14 August 2017.

ADDRESS For CORRESPONDENCE: Prof. Dongfeng Zhang, School of Public Health, Qingdao University, No. 38 Dengzhou Road, Shibei District, Qingdao, Shandong 266021, China. E-mail: zhangdongfeng@qdu.edu.cn 
possibly causing hazardous or dangerous situations. Agingrelated hearing impairment usually starts at high frequencies and then progresses to the lower frequencies as people age. High-frequency warning sounds - for example, turning signals, escaping steam, and beepers - are not heard or localized when hearing loss occurs, which could potentially cause disastrous consequences (Gates \& Mills, 2005). Many elderly people often complain that they can hear but cannot understand because of hearing loss. Besides the performance of the auditory system, cognitive function is also an important determinant for retaining quality of life and living independently for middle- and older-aged people (Harris \& Deary, 2011).

Epidemiological studies have indicated that hearing and cognitive functions are closely associated with each other in general populations (Schmulian Taljaard et al., 2015). There may be an underlying mechanistic link that regulates the relationship between hearing and cognitive function (Wayne \& Johnsrude, 2015). Here, we hypothesize that the correlation between hearing and cognitive function could be due to genes and/or environment. In the literature, no studies about genetic and/or environmental correlations between hearing and cognitive functions have been found. A number of studies have suggested that the hearing and cognitive function are affected by both genetic and environmental factors using twin or family methods (DeStefano et al., 2003; Hendrickx et al., 2013; Karlsson et al., 1997; Momi et al., 2015; Polderman et al., 2015; Viljanen et al., 2007; Wingfield et al., 2007). Although the genetic influences on hearing and cognitive function have been well studied in Western populations, the genetic background for their concordance remains unclear. This article first reports results from the first twin study on hearing and cognitive performance in Chinese middle- and older-aged adult twins as part of a comprehensive investigation on genetic and environmental regulation on aging-related phenotypes or traits; and second, presents our novel findings on the genetic and environmental bases for the correlation between agingrelated hearing and cognitive performances. The findings may have an insight into the genetic basis for hearing and cognition and may also reveal at least the etiology of hearing and cognition.

\section{Materials and Methods}

\section{Sample Collection}

The sample collection was conducted through the Qingdao Twin Registry (QTR), the first twin registry in China (Li et al., 2006; 2013), established at the Qingdao Center for Disease Control and Prevention (Qingdao CDC; Duan et al., 2013; Pang et al., 2006). We recruited the twin samples through the QTR and local CDC, and via community and media publicity from 2012 to 2013 . The co-twins suffering from auditory or cognitive disease, and incomplete twin pairs were excluded from the study. We sampled a total of
379 complete adult twin pairs, including 240 monozygotic (MZ) pairs (114 male or MZM and 126 female MZF pairs) and 139 dizygotic (DZ) pairs (41 DZM, 39 DZF, 59 opposite sex or OSDZ pairs) aged $51.8 \pm 7.6$ (40-80 years old). The number of participants by educational level categorized by non-schooling, primary school, middle school, high middle school, college, and above were $39,130,342,181$, and 76 , accounting for $5.1 \%, 16.9 \%, 44.5 \%, 23.6 \%$, and $9.9 \%$, respectively. The collected twin samples did not represent a specific social class or educational status. Zygosity was identified using DNA markers at the laboratory of Qingdao Blood Center, with 99.9\% correct assignment (Becker et al., 1997; Tomsey et al., 2001).

The study was approved by Regional Ethics Committee at Qingdao CDC Institutional Review Boards (QDCDCIRB) and conducted according to the principles of the Helsinki Declaration. All participants signed a consent form and completed the questionnaire and pure-tone audiometry test at a community hospital or the local service center of the Qingdao CDC.

\section{Measurements of Cognitive Function}

The Montreal Cognitive Assessment (MoCA, www. mocatest.org) was used to evaluate the cognitive function of the participants. MoCA, including several domains of cognitive function, is a very good age-sensitive and effective tool for cognitive screening (Gluhm et al., 2013; Luis et al., 2009; Nasreddine et al., 2005). Each twin pair was interviewed face-to-face and evaluated by the same well-trained investigator.

\section{Measurements of Hearing}

The most common sound frequencies in the living and working environment are in the $0.5-8 \mathrm{kHz}$ range. The air-conducted, pure-tone hearing threshold levels at low $(0.5$ and $1 \mathrm{kHz}$ ), middle ( 2 and $4 \mathrm{kHz}$ ), and high ( 8 and $12.5 \mathrm{kHz}$ ) frequencies were tested by the standard audiometer on both ears of every subject. The same audiologist performed the audiometric measurements in a sound-isolated room using a clinical audiometer (EN.60645-1,-2type:2A. Madsem Iteral 2; GN otometrics, Denmark) according to the standard procedure. The audiometer was calibrated every morning before measurement.

The hearing threshold value (in decibels, $\mathrm{dB}$ ) indicates the smallest sound intensity heard by the participant. The lower threshold of the two ears of each participant was recorded for use in the study. The lower hearing threshold used in the study meant a better hearing function.

\section{Data Analysis}

Epidata3.1 (www.epidata.dk) was used to input and correct the data. Because the distributions of all indicators were skewed, a Box-Cox transformation was applied to ensure normal or approximately normal distribution. The BoxCox transformation was done using the free $\mathrm{R}$ package car 
TABLE 1

Characteristics of the Sample

\begin{tabular}{lllllll}
\hline & \multicolumn{5}{c}{ Educational level } & \\
\cline { 2 - 6 } Age (year) & Non-schooling & Primary school & Middle school & High middle school & College and above & Total (\%) \\
\hline $40-45$ & 0 & 22 & 65 & 28 & 27 & $142(18.7)$ \\
$45-50$ & 3 & 37 & 101 & 45 & 16 & $202(26.6)$ \\
$50-55$ & 3 & 14 & 52 & 64 & 11 & $144(19.0)$ \\
$55-60$ & 13 & 14 & 82 & 26 & 15 & $150(19.8)$ \\
$60-65$ & 10 & 26 & 26 & 11 & 5 & $78(10.3)$ \\
$65-70$ & 4 & 11 & 6 & 2 & 1 & $24(3.2)$ \\
$70-80$ & 6 & 6 & 4 & 1 & 1 & $18(2.4)$ \\
Total (\%) & $39(5.1)$ & $130(17.2)$ & $336(44.3)$ & $177(23.4)$ & $76(10.0)$ & $758(100.0)$ \\
\hline
\end{tabular}

TABLE 2

Descriptive Statistics and Linear Regression for Covariates

\begin{tabular}{|c|c|c|c|c|c|c|c|c|c|c|}
\hline \multirow[b]{2}{*}{ Indicator } & \multirow[b]{2}{*}{ Median } & \multirow[b]{2}{*}{$95 \%$ Range } & \multirow[b]{2}{*}{$n_{M Z}$ (pairs) } & \multirow[b]{2}{*}{$n_{D Z}$ (pairs) } & \multicolumn{2}{|c|}{ Age } & \multicolumn{2}{|l|}{ Sex } & \multicolumn{2}{|c|}{ Age $\times$ sex } \\
\hline & & & & & Coefficients & $p$ & Coefficients & $p$ & Coefficients & $p$ \\
\hline Hearing: $0.5 \mathrm{kHz}$ & 20 & $5-40$ & 225 & 131 & 0.277 & .057 & 2.409 & .636 & -0.046 & .641 \\
\hline Hearing: $1 \mathrm{kHz}$ & 15 & $0-35$ & 225 & 131 & 0.327 & .024 & 1.787 & .725 & -0.053 & .588 \\
\hline Hearing: 2 kHz & 10 & $0-40$ & 224 & 131 & 0.640 & $7.974 \times 10^{-5}$ & 7.340 & .197 & -0.173 & .113 \\
\hline Hearing: $4 \mathrm{kHz}$ & 15 & $0-64$ & 224 & 131 & 0.745 & .004 & -9.467 & .299 & -0.089 & .611 \\
\hline Hearing: 8 kHz & 20 & $0-75$ & 222 & 130 & 1.058 & .001 & -11.133 & .294 & -0.015 & .941 \\
\hline Hearing: $12.5 \mathrm{kHz}$ & 50 & $20-75$ & 84 & 39 & 1.781 & .595 & -122.617 & .265 & 2.058 & .369 \\
\hline Cognitive function & 22 & $8.2-28$ & 240 & 139 & -4.4963 & .001 & -9.1709 & .842 & 0.495 & .575 \\
\hline
\end{tabular}

Note: males coded 1 and females coded 2.

(http://cran.r-project.org/web/packages/car/index.html).

To fit the twin models, age, gender, and their interaction were considered as covariates to adjust their influences on all indices. Twin correlation was measured by calculating the intra-class coefficients (ICCs) and compared between $\mathrm{MZ}$ and DZ. ICCs were estimated using the R package mets (http://cran.r-project.org/web/packages/mets/index.html) adjusting the effects of age, sex, and their interaction.

\section{Twin Modeling}

The bivariate twin modeling was adopted to dissect the correlation between hearing and cognitive function to genetic $\left(r_{\mathrm{G}}\right)$, common environmental $\left(r_{\mathrm{C}}\right)$, and unique environmental correlation $\left(r_{\mathrm{E}}\right)$, to be indicated by the full ACE model. The nested models, including AE, CE, and AC, were fitted by dropping every component whose statistical significance was tested by the likelihood ratio test. The full and the nested models were compared so as to choose the best-fitting model on the basis of the parsimonious principle and the Akaike's Information Criterion (AIC; Akaike, 1987) with the lowest value for the best-fitting model. The cross-twin, cross-trait correlation (CTCTC) was estimated before fitting the bivariate model and compared across zygosities to provide indication of genetic mediation between the two traits of interest. Twin models were fitted by R package mets and Mx (http://www.vcu.edu/mx).

\section{Results}

The distribution of age and educational levels of the twin sample are presented in Table 1 . The subjects were mainly between 40 and 65 years old, accounting for about $94.5 \%$ of the participants. The educational levels of the participants were mainly from primary school, middle school, and high middle school, accounting for about $84.8 \%$ of the participants. The descriptive statistics, including median, percentiles, and the fitted linear models on each measurement for the effects of age, sex, and the interaction, are shown in Table 2. There were increasing patterns of hearing thresholds at $1 \mathrm{kHz}, 2 \mathrm{kHz}, 4 \mathrm{kHz}$, and $8 \mathrm{kHz}$ frequencies and a declining pattern of cognitive function with increasing age as indicated by the significantly positive and negative regression coefficients respectively $(p<.05)$ except for $0.5 \mathrm{kHz}$ of hearing, which was at borderline significance. Sex differences (males coded 1 and females 2) and age-sex interactions for all indicators were observed to be insignificant $(p>.05)$.

The phenotypic correlations between cognitive and hearing functions at $0.5 \mathrm{kHz}, 1 \mathrm{kHz}, 2 \mathrm{kHz}, 4 \mathrm{kHz}, 8 \mathrm{kHz}$, and $12.5 \mathrm{kHz}$ in the whole sample were $-0.26,-0.24$, $-0.21,-0.22,-0.26$, and -0.06 , respectively. The CTCTCs for six combinations were higher in $\mathrm{MZ}$ than those of $\mathrm{DZ}$ twins, implying potentially genetic involvement in the correlations between hearing and cognition (Table 3) although the CTCTCs were generally low to mild in both MZ and DZ twin pairs. Based on the results for CTCTCs, we additionally fitted bivariate twin models to estimate the genetic and environmental correlations for cognition with the six levels of hearing. Besides the full models, the nested models were fitted by dropping the genetic and/or environmental components in the covariance between hearing and cognitive function so as to select the best-fitting models. In Table 4, 
TABLE 3

Phenotypic Correlation Between Hearing and Cognitive Function Pair

\begin{tabular}{llllll}
\hline & \multicolumn{5}{c}{ CTCTC } \\
\cline { 2 - 6 } Hearing and cognitive function pair & $\mathrm{MZ}$ & $n_{\mathrm{MZ}}$ & $\mathrm{DZ}$ & $n_{\mathrm{DZ}}$ & $\mathrm{p}$ \\
\hline $0.5 \mathrm{kHz}$ : Cognition & -0.30 & 225 & -0.17 & 133 & .212 \\
$1 \mathrm{kHz}$ : Cognition & -0.25 & 225 & -0.12 & 133 & .222 \\
$2 \mathrm{kHz}$ : Cognition & -0.26 & 225 & -0.07 & 133 & .075 \\
$4 \mathrm{kHz}$ : Cognition & -0.26 & 225 & -0.01 & 133 & $.020^{*}$ \\
$8 \mathrm{kHz}$ : Cognition & -0.32 & 223 & -0.15 & 132 & .104 \\
$12.5 \mathrm{kHz}$ : Cognition & -0.17 & 106 & 0.05 & 66 & .166 \\
\hline
\end{tabular}

Note: CTCTC $=$ cross-twin cross-trait correlation. ${ }^{*} p<.05$.

TABLE 4

Estimated Genetic and Environmental Correlation Between Hearing and Cognitive Function

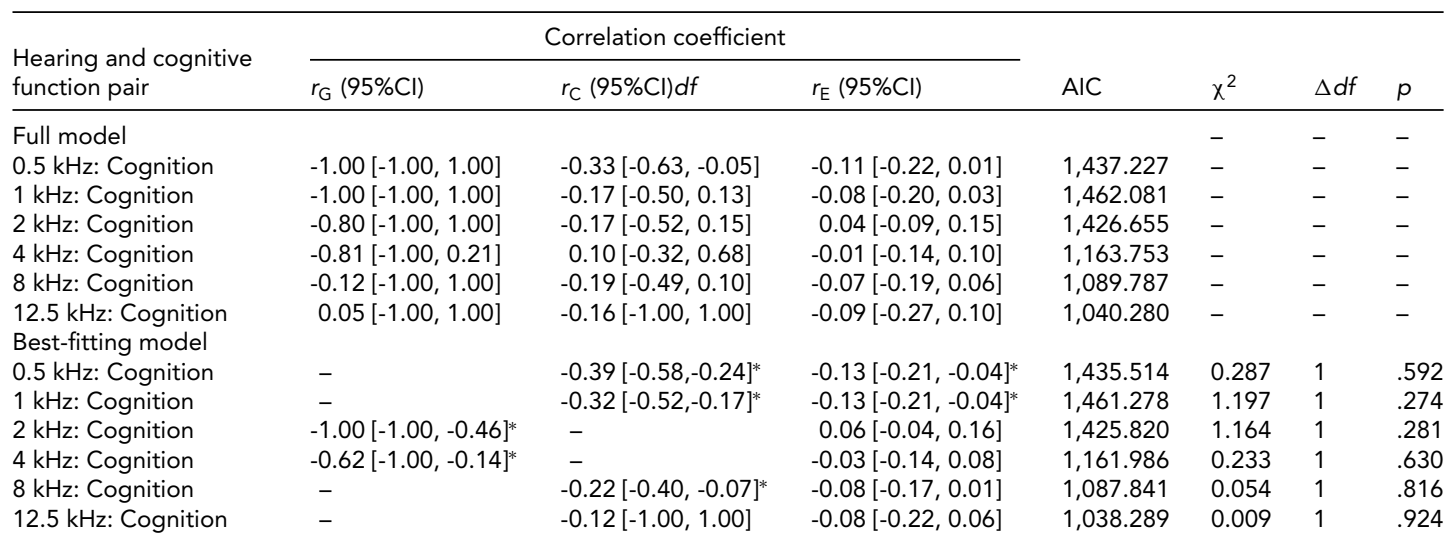

Note: $r_{G}$, genetic correlation between traits; $r_{C}$, shared environmental correlation between traits; $r_{E}$, non-shared environmental correlation between traits. Akaike's information criterion, $\chi^{2}=$ difference of $\chi^{2}$ value, $\Delta d f=$ change in degree of freedom; $p=\chi^{2}$ test in model fitting. The best-fitting model was chosen on the basis of a change in $\chi^{2}$ not representing a significant worsening of fit. ${ }^{*} p<.05$.

we show the estimated genetic and environmental correlations in the full and the best-fitting models for the combination of hearing and cognitive function. The correlations between middle frequencies of hearing, such as $2 \mathrm{kHz}$ as well as $4 \mathrm{kHz}$, and cognition were best fitted by the AE models. However, the correlations of low as well as high frequencies of hearing with cognition had the CE model as the best-fitting model. We observed there existed moderate to high genetic correlations $\left(r_{\mathrm{G}}\right)$ between middle frequencies of hearing and cognitive function, all with 95\% CIs beyond zero. The genetic correlation between $2 \mathrm{kHz}$ and cognition was $r_{\mathrm{G}}=-1.00,95 \% \mathrm{CI}[-1.00,-0.46]$, and between $4 \mathrm{kHz}$ and cognition it was $r_{\mathrm{G}}=-0.62,95 \% \mathrm{CI}[-1.00,-0.14]$, revealing the genetic mediation between the two measurements. We did not find significant genetic correlations between low as well as high frequencies of hearing and cognition in the study. Instead, we found significant common and unique environmental correlations between cognition and low frequencies of hearing at $0.5 \mathrm{kHz}$ and $1 \mathrm{kHz}(-0.13$ to -0.39$)$ as well as common environmental correlation between $8 \mathrm{kHz}$ and cognition $(-0.22)$, all with 95\% CIs well beyond zero.

\section{Discussion}

We conducted twin modeling to dissect the genetic and environment components in the correlations between hearing and cognitive function in Chinese adult twins without major hearing and cognitive difficulties. The results from our sizable sample gave a reliable and valid assessment of hearing and cognitive function.

The genetic correlation expresses the extent to which two measurements are mediated by the same set of genes. Studying genetic correlations can facilitate exploration of pleiotropic genes or genetic variants. Our results indicated that the correlations between hearing and cognitive function originated not only from genetic but also environmental causes. The bivariate genetic analysis implied that the correlation between middle frequencies of hearing and cognition stemmed predominantly from genetic rather than environmental factors, although in the opposite direction, which was in line with the corresponding CTCTC and covariance estimate, it suggested that the underlying genes may have pleiotropic effects on both traits. However, the correlations between cognition and low as well as high frequencies of hearing were mainly derived from common 
and unique environmental causes but with negative value, meaning that they may share similar environmental factors but with opposite effects. All the significant shared genetic, common, and unique environmental factors may lead to better or worse hearing and cognitive functional status simultaneously although the correlation coefficients were negative.

The present results suggested that the highly significant correlations of middle frequencies of hearing with cognition could be more of a genetic nature and worth further exploration by molecular genetics approaches. Further studies based on high-throughput techniques, such as next-generation sequencing of DNA could help to find genetic variants associated with our observed correlations and provide biological basis for the genetic pleiotropy between hearing and cognition.

Finally, we would like to state the limitations of the study. First, the age of the twin samples were relatively young compared with Western studies and covered a large age span (40-80 years). The non-linear effect of age-dependent change in hearing and cognition, if any, could have been averaged out, which could possibly contribute to reducing the power of the study, although we adjusted for the effect of age in twin modeling. Second, the sample size was still limited, which can be responsible for the wide $95 \%$ confidence intervals, as shown in Table 4. Third, MoCA is just a screening instrument, so it may not be optimally suitable for assessing the participants' cognitive function, particularly for the younger samples. Well-defined future studies should help to verify our findings and provide more reliable assessment on the genetic pleiotropy for hearing and cognition during aging.

\section{Acknowledgment}

This study was supported by National Natural Science Foundation of China (31371024) and the Qingdao Postdoctoral Application Research Project (2016058).

\section{Disclosure of Interests}

None.

\section{References}

Akaike, H. (1987). Factor analysis and AIC. Psychometrika, 52, 317-332.

Becker, A., Busjahn, A., Faulhaber, H. D., Bahring, S., Robertson, J., Schuster, H., \& Luft, F. C. (1997). Twin zygosity. Automated determination with microsatellites. Journal of Reproductive Medicine, 42, 260-266.

DeStefano, A. L., Gates, G. A., Heard-Costa, N., Myers, R. H., \& Baldwin, C. T. (2003). Genomewide linkage analysis to presbycusis in the Framingham heart study. Archives of Otolaryngology-Head \& Neck Surgery, 129, 285-289.
Duan, H., Ning, F., Zhang, D., Wang, S., Zhang, D., Tan, Q., ... Pang, Z. (2013). The Qingdao twin registry: A status update. Twin Research and Human Genetics, 16, 79-85.

Gates, G. A., \& Mills, J. H. (2005). Presbycusis. Lancet, 366, 1111-1120.

Gluhm, S., Goldstein, J., Loc, K., Colt, A., Liew, C. V., \& CoreyBloom, J. (2013). Cognitive performance on the minimental state examination and the Montreal cognitive assessment across the healthy adult lifespan. Cognitive and Behavioral Neurology, 26, 1-5.

Harris, S. E., \& Deary, I. J. (2011). The genetics of cognitive ability and cognitive ageing in healthy older people. Trends in Cognitive Sciences, 15, 388-394.

Hendrickx, J. J., Huyghe, J. R., Topsakal, V., Demeester, K., Wienker, T. F., Laer, L. V., ... de Heyning, P. V. (2013). Familial aggregation of pure tone hearing thresholds in an aging European population. Otology \& Neurotology, 34, 838844.

Huang, Q., \& Tang, J. (2010). Age-related hearing loss or presbycusis. European Archives of Oto-rhino-laryngology, 267, 1179-1191.

Karlsson, K. K., Harris, J. R., \& Svartengren, M. (1997). Description and primary results from an audiometric study of male twins. Ear and Hearing, 18, 114-120.

Li, L., Gao, W., Lv, J., Cao, W., Zhan, S., Yang, H., \& Hu, Y. (2006). Current status of the Chinese national twin registry. Twin Research and Human Genetics, 9, 747-752.

Li, L., Gao, W., Yu, C., Lv, J., Cao, W., Zhan, S., ... Hu, Y. (2013). The Chinese national twin registry: an update. Twin Research and Human Genetics, 16, 86-90.

Luis, C. A., Keegan, A. P., \& Mullan, M. (2009). Cross validation of the Montreal cognitive assessment in community dwelling older adults residing in the Southeastern US. International Journal of Geriatric Psychiatry, 24, 197-201.

Momi, S. K., Wolber, L. E., Fabiane, S. M., MacGregor, A. J., \& Williams, F. M. (2015). Genetic and environmental factors in age-related hearing impairment. Twin Research and $\mathrm{Hu}$ man Genetics, 18, 383-392.

Nasreddine, Z. S., Phillips, N. A., Bedirian, V., Charbonneau, S., Whitehead, V., Collin, I., ... Chertkow, H. (2005). The Montreal Cognitive Assessment, MoCA: a brief screening tool for mild cognitive impairment. Journal of the American Griatrics Society, 53, 695-699.

Pang, Z., Ning, F., Unger, J., Johnson, C. A., Wang, S., Guo, Q., ... Lee, L. (2006). The Qingdao twin registry: A focus on chronic disease research. Twin Research and Human Genetics, 9, 758-762.

Polderman, T. J., Benyamin, B., de Leeuw, C. A., Sullivan, P. F., van Bochoven, A., Visscher, P. M., \& Posthuma, D. (2015). Meta-analysis of the heritability of human traits based on fifty years of twin studies. Nature Genetics, 47, 702-709.

Schmulian Taljaard, D., Olaithe, M., Brennan-Jones, C. G., Eikelboom, R. H., \& Bucks, R.S. (2015). The relationship between hearing impairment and cognitive function: A meta-analysis in adults. Clinical Otolaryngology, 41, 718-729. 
Tomsey, C. S., Kurtz, M., Kist, F., Hockensmith, M., \& Call, P. (2001). Comparison of PowerPlex 16, PowerPlex1.1/2.1, and ABI AmpfISTR Profiler Plus/COfiler for forensic use. Croatian Medical Journal, 42, 239-243.

Viljanen, A., Kaprio, J., Pyykko, I., Sorri, M., Kauppinen, M., Koskenvuo, M., \& Rantanen, T. (2007). Genetic and environmental influences on hearing at different frequencies separately for the better and worse hearing ear in older women. International Journal of Audiology, 46, 772-779.
Wayne, R. V., \& Johnsrude, I. S. (2015). A review of causal mechanisms underlying the link between age-related hearing loss and cognitive decline. Ageing Research Reviews, 23(Pt B), 154-166.

Wingfield, A., Panizzon, M., Grant, M. D., Toomey, R., Kremen, W. S., Franz, C. E., ... Lyons, M. (2007). A twinstudy of genetic contributions to hearing acuity in late middle age. Journals of Gerontology Series A-Biological Sciences and Medical Sciences, 62, 1294-1299. 